\title{
Modelling of metabolic control by Short Chain Fatty Acids at the level of the functional proteomic analysis
}

\author{
L. E. Sánchez-Mejorada Zúñiga ${ }^{1,2}$, Joanne Connolly ${ }^{3}$, Kelly McMahon $^{3}$, C. A. Evans ${ }^{2}$, \\ and B. M. Corfe \\ ${ }^{1}$ Molecular Gastroenterology Research Group, Academic Unit of Surgical Oncology, Department of Oncology, The Medical \\ School, Beech Hill Road, Sheffield, S10 2RX, ${ }^{2}$ Biological and Systems Engineering Group, ChELSI Institute, Department of \\ Chemical and Biological Engineering, University of Sheffield, Sheffield, S1 3JD and ${ }^{3}$ Waters Corporation, Floats Rd, \\ Manchester, Lancashire M23 9LZ, UK
}

Dietary residues are fermented in the large intestine to produce short-chain fatty acids (SCFAs) in the colon ${ }^{(1,2,3)}$. SCFAs, particularly butyrate, inhibit histone deacetylation, may be chemopreventive, and regulate acetylation of metabolic pathways ${ }^{(4,5,6,7,8)}$. As metabolic pathways are subject of being regulated by acetylation ${ }^{(9)}$, it is possible to model and elucidate the interaction among the diverse metabolic pathways and their acetylation patterns.

Mitochondrially enriched fractions from HCT116 colon cancer cells treated with $10 \mathrm{mM}$ propionate and/or butyrate in a $2 \times 2$ factorial design with two independent repeats were analysed using a label-free workflow. The total proteome and acetylated proteome were identified and the data interrogated qualitatively as a first step using ProteinLynxGlobal Server and Scaffold software packages. Representation analysis was undertaken DAVID and Reactome. Enzymes for major metabolic pathways involving acetyl-CoA (as a component: Glycolysis, Tricarboxylic Acid, Pyruvate metabolism and $\beta$-oxidation) were searched for by Uniprot identifier.

Representation analysis indicated translation pathways were particularly enriched in the global proteome $\left(P=1.6 \times 10^{-59}\right)$ suggesting presence of ribosomes in the fractions. All metabolic pathways were represented with $65 \%$ coverage for Glycolysis, $81 \%$ for TCA, and $55 \%$ for $\beta$-oxidation. The acetylated proteome was further enriched for translation $\left(P=1.6 \times 10^{-3}\right)$. All metabolic pathways were represented in the acetylated proteome with $23 \%$ for Glycolysis, $19 \%$ for TCA, and $18 \%$ for $\beta$-oxidation. Principal Components Analysis (PCA) showed good clustering of replicates and a marked difference between the effects of propionate and butyrate.

The data suggest that the translational apparatus is highly acetylated and therefore may represent an additional level of functional control by SCFA related to transcriptions in cellular programming. The PCA data confirm our published findings that butyrate and propionate have distinct rather than graded cellular effects. Datamining is underway to establish whether spectra for acetylpeptides in the metabolic enzymes exhibit altered counts after treatments.

1. Lupton J (2000) Nutrition 16, 558/561.

2. Scheppach W, Bartram H, Richter F et al. (1995) Eur J Cancer 31A, 1077-1080.

3. Hague A, Butt A and Paraskeva C (1996) Proc Nut Soc 55, 937-943.

4. Candido E, Reeves R and Davies J (1978) Cell 14, 105-113.

5. Lea M and Randolph V (1998) Anticancer Res 18, 2717-2722.

6. Donohoe D, Collins L, Wali A et al. (2012) Mol Cell 48, 612-626.

7. Bingham S (1990) Proc Nutr Soc 49, 153-171.

8. Jahns F, Wilhelm A, Jablonowski N et al. (2011) Carcinogenesis 32, 913-920

9. Xiong Y, Lei Q, Zhao S et al. (2011) Cold Spring Harb Symp Quant Biol 76, 285-289. 